\title{
Reduced Routley-Meyer semantics for the logics characterized by natural implicative expansions of Kleene's strong 3-valued matrix
}

\author{
Gemma Robles \\ Dpto. de Psicología, Sociología y Filosofía, Universidad de León \\ Campus de Vegazana, s/n, 24071, León, Spain \\ gemma.robles@unileon.es; http://grobv.unileon.es
}

\begin{abstract}
The aim of this paper is to provide a reduced Routley-Meyer semantics for the logics characterized by all natural implicative expansions of Kleene's strong 3-valued matrix (with two designated values, as well as with only one) susceptible to be interpreted in Routley-Meyer semantics.

Keywords: Routley-Meyer semantics; Kleene's strong 3-valued matrix; natural conditionals; 3 -valued logics; substructural logics.
\end{abstract}

\section{Introduction}

Given a matrix semantics, a conditional is natural if it fulfills the three following conditions: (1) it coincides with the classical conditional when restricted to the classical values $T$ and $F$; (2) it satisfies the Modus Ponens; and (3) it is assigned a designated value when the value assigned to its antecedent is less than or equal to the value assigned to its consequent (cf. [18]). In [14] and [15], it is presented a "bivalent" Belnap-Dunn semantics (cf. [3], [4], [7] and [8]) for all natural implicative expansions of Kleene's strong 3-valued matrix (cf. [10]) with two designated values (cf. [14]) as well as with only one designated value (cf. [15]). Well then, the aim of this paper is to provide a Routley-Meyer semantics for the logics characterized by all natural implicative expansions of Kleene's strong 3 -valued matrix (with both only one and also two designated values) susceptible to be interpreted in this type of semantics.

Routley-Meyer type ternary relational semantics (RM-semantics) was introduced in the early 70s of the past century (cf. [5], [16] and references therein). It was particularly defined for interpreting relevance logics, but it was soon noticed that an ample class of logics not belonging to the relevance logics family could also be characterized by this semantics. RM-semantics is a relational type semantics. It can be distinguished from standard Kripke semantics in two aspects: on the one hand, the accessibility relation between worlds (points, setups or whatever the name is preferred) is a ternary relation instead of a binary 
one and, on the other hand, negation formulas are interpreted by the "Routley operator" (or "Routley star") in each possible world w.r.t. its so-called "starimage world", instead of being interpreted in each possible world in function of the argument's value of the negation formula in that same possible world.

There are essentially two types of RM-semantics: (1) RM-semantics with a set of designated points w.r.t. which validity of formulas is decided $\left(\mathrm{RM}_{1^{-}}\right.$ semantics) and (2) RM-semantics without a set of designated points, where validity of formulas is decided w.r.t. the set of all points ( $\mathrm{RM}_{0}$-semantics). These two type of semantics can also be found in standard Kripke semantics. As for $\mathrm{RM}_{1}$-semantics, we have reduced $\mathrm{RM}_{1}$-semantics, where the set of designated points is reduced to a singleton, and unreduced $\mathrm{RM}_{1}$-semantics.

It is to be remarked that it is not possible to give an RM-semantics to logics weaker than (not containing) Sylvan and Plumwood's minimal logic $\mathrm{B}_{\mathrm{M}}$ (cf. [17]). Therefore, the aim of this paper is to provide a reduced $\mathrm{RM}_{1}$-semantics for the logics characterized by all natural implicative expansions of Kleene's strong 3-valued matrix (with both one and also two designated values), which are representable in RM-semantics (i.e., logics containing $\mathrm{B}_{\mathrm{M}}$ ).

The present paper pursues previous work by the author on RM-semantics for 3-valued logics. In [12], a reduced $\mathrm{RM}_{0}$-semantics for Łukasiewicz's 3-valued logic 3 is presented, whereas the same type of semantics is given for both Gödelian 3-valued logic G3 and its paraconsistent variant G3Ł in [11]. Nevertheless, and in addition to the fact that more basic 3-valued logics are given an RM-semantics, the results here presented suppose an advance w.r.t. those just recalled in the two following aspects. (1) Generality: all the logics investigated are interpreted from a unified and general point of view ( $\mathrm{L} 3$ and $\mathrm{G} 3_{\mathrm{E}}$ are to be found among these logics); (2) the type of RM-semantics used in the present paper: on the one hand, $\mathrm{RM}_{1}$-semantics (the RM-semantics used in what follows) is more general than $\mathrm{RM}_{0}$-semantics in the sense that in most significant cases the latter can be defined from the former, but not conversely. On the other hand, reduced RM-semantics are preferable to unreduced RM-semantics when it is possible to define the former (cf. [5], [16]). (In this second aspect, the RMsemantics developed in this paper is similar (but more general) to the reduced $\mathrm{RM}_{1}$-semantics supplied for Brady's 4-valued logic of the relevant conditional BN4 and its accompanying 4-valued logic (of relevant) entailment E4 in [13].)

The structure of the paper is as follows. In $\S 2$, we firstly state some preliminary basic notions as used in the paper. Then, Kleene's strong 3-valued matrix MK3 (our label) is defined. In $\S 3$, the notion of a "natural conditional" (according to [18]) is introduced and all natural implicative expansions of MK3 (with both one and also two designated values), defined in [14] and [15], are recalled (we remark that there are stricter notions of "natural" in the literature; cf., e.g., [2]). It develops that only six of these implicative expansions of MK3 verify all axioms and rules of Sylvan and Plumwood's minimal logic $B_{M}$. Consequently, only the six logics (let us name them Lt $i$-logics) determined by each one of the aforementioned six implicative expansions of MK3 can be given an RM-semantics. In $\S 4$, the six Lt $i$-logics are defined in a general and unified way as extensions of Routley and Meyer's disjunctive basic logic B (cf. [16], 
Chapter 4). In $\S 5$, a reduced $\mathrm{RM}_{1}$-semantics is provided for each one of the six Lt $i$-logics and the (strong) soundness theorems are proved. In $\S 6$, the extension and primeness lemmas are noted (cf. [16] and also [6]). In $\S 7$, we prove a series of preliminary lemmas to the completeness theorem. Finally, in $\S 8$, the (strong) completeness theorems are proved. We have added three appendices: Appendix 1 , on the natural implicative expansions of MK3, and Appendices 2 and 3, on the Lti-logics.

\section{Kleene's strong 3-valued matrix}

In this section, Kleene's strong 3-valued matrix is recalled. Firstly, we state some preliminary definitions where we recall some basic notions as used in the present paper. Then, Kleene's strong 3-valued matrix is defined.

Definition 2.1 (Language) The propositional language consists of a denumerable set of propositional variables $p_{0}, p_{1}, \ldots, p_{n}, .$. , and the following connectives $\rightarrow$ (conditional), $\wedge$ (conjunction), $\vee$ (disjunction), $\neg$ (negation). The biconditional $(\leftrightarrow)$ and the set of wffs are defined in the customary way. A, B etc. are metalinguistic variables.

Definition 2.2 (Logics) A logic $L$ is a structure $\left(\mathcal{L}, \vdash_{L}\right)$ where $\mathcal{L}$ is a propositional language and $\vdash_{L}$ is a (proof-theoretical) consequence relation defined on $\mathcal{L}$ by a set of axioms and a set of rules of derivation. The notions of 'proof' and 'theorem' are understood as it is customary in Hilbert-style axiomatic systems $\left(\Gamma \vdash_{L} A\right.$ means that $A$ is derivable from the set of wffs $\Gamma$ in $L$; and $\vdash_{L}$ A means that $A$ is a theorem of $L$ ).

Definition 2.3 (Extensions and expansions) Let $L$ be a logic formulated with axioms $a 1, \ldots$, an and rules of derivation $r 1, \ldots, r m$. A logic $L^{\prime}$ includes $L$ iff $a 1, \ldots$, an are theorems of $L^{\prime}$ and rules $r 1, \ldots, r m$ are provable in $L^{\prime}$. We shall generally refer to logics including $L$ by EL-logics. Notice that an EL-logic can be an extension of $L$ (a strengthening of $L$ in the language of $L$ ) or an expansion of it (a strengthening of $L$ in an expansion of the language of $L$ ). An extension $L^{\prime}$ of $L$ is a proper extension if $L^{\prime}$ is not included in $L$.

Definition 2.4 (Logical matrix) A (logical) matrix is a structure $(\mathcal{V}, D, \mathrm{~F})$ where (1) $\mathcal{V}$ is a (ordered) set of (truth) values; (2) $D$ is a non-empty proper subset of $\mathcal{V}$ (the set of designated values); and (3) $\mathrm{F}$ is the set of $n$-ary functions on $\mathcal{V}$ such that for each n-ary connective $c$ (of the propositional language in question), there is a function $f_{c} \in \mathrm{F}$ such that $\mathcal{V}^{n} \rightarrow \mathcal{V}$.

Definition 2.5 (M-interpretation, M-consequence, M-validity) Let $M$ be a matrix for (a propositional language) $\mathcal{L}$. An M-interpretation $I$ is a function from $\mathcal{F}$ to $\mathcal{V}$ according to the functions in $\mathrm{F}$. Then, for any set of wffs $\Gamma$ and wff $A, \Gamma \vDash_{M} A$ ( $A$ is a consequence of $\Gamma$ according to $M$ ) iff $I(A) \in D$ whenever 
$I(\Gamma) \in D$ for all $M$-interpretations $I(I(\Gamma) \in D$ iff $I(B) \in D$ for each $B \in \Gamma)$. In particular, $\vDash_{M} A$ ( $A$ is $M$-valid; $A$ is valid in the matrix $M$ ) iff $I(A) \in D$ for all $M$-interpretations $I .\left(B y \vDash_{M}\right.$ we shall refer to the relation defined in $\left.M\right)$.

Definition 2.6 (Kleene's strong 3-valued matrix) The propositional language consists of the connectives $\wedge, \vee, \neg$. Kleene's strong 3-valued matrix, MK3 (our label), is the structure $(\mathcal{V}, D, F)$ where (1) $\mathcal{V}=\{0,1,2\}$ and it is ordered as shown in the following diagram

$$
\left.\right|_{0} ^{2}
$$

(2) $D=\{1,2\}$ or $D=\{2\}$; (3) $\mathrm{F}=\left\{f_{\wedge}, f_{\vee}, f_{\neg}\right\}$ where $f_{\wedge}$ and $f_{\vee}$ are defined as the glb (or lattice meet) and the lub (or lattice joint), respectively, $f_{\neg}$ is an involution with $f_{\neg}(2)=0, f_{\neg}(0)=2$ and $f_{\neg}(1)=1$. We display the tables for $\wedge, \vee$ and $\neg$ :

\begin{tabular}{|c|c|c|c|c|c|c|c|c|c|}
\hline$\wedge$ & 0 & 1 & 2 & $V$ & 0 & 1 & 2 & $\neg$ & 0 \\
\hline 0 & 0 & 0 & 0 & 0 & 0 & 1 & 2 & 0 & 2 \\
\hline 1 & 0 & 1 & 1 & 1 & 1 & 1 & 2 & 1 & 1 \\
\hline 2 & 0 & 1 & 2 & 2 & 2 & 2 & 2 & 2 & 0 \\
\hline
\end{tabular}

The notions of an MK3-interpretation, MK3-consequence and MK3-validity are defined according to the general Definition 2.5.

The notion of a logic determined by a given matrix can be understood as stated in the following definition.

Definition 2.7 (Logics determined by matrices) Let $\mathcal{L}$ be a propositional language, $M$ a matrix for $\mathcal{L}$ and $\vdash_{L}$ a (proof theoretical) consequence relation defined on $\mathcal{L}$. Then, the logic $L$ (cf. Definition 2.2) is determined by $M$ iff for every set of wffs $\Gamma$ and wff $A, \Gamma \vdash_{L} A$ iff $\Gamma \vDash_{M} A$. In particular, the logic $L$ (considered as the set of its theorems) is determined by $M$ iff for every wff $A$, $\vdash_{L} A$ iff $\vDash_{M} A$ (cf. Definition 2.5).

The logic determined by MK3 can be named here $\mathrm{K} 3^{1}$ (only one designated value) or $\mathrm{K}^{2}$ (two designated values) (cf. [8], $§ 3.4$ on these logics).

\section{Natural implicative expansions of MK3}

Following Tomova [18], we define "natural conditionals" as follows. 
Definition 3.1 (Natural conditionals) Let $\mathcal{L}$ be a propositional language with $\rightarrow$ among its connectives and $M$ be a matrix for $\mathcal{L}$ where the values $x$ and $y$ represent the supremum and the infimum in $\mathcal{V}$. Then, an $f_{\rightarrow-\text { function on } \mathcal{V} \text { defines }}$ a natural conditional if the following conditions are satisfied:

1. $f_{\rightarrow}$ coincides with (the $f_{\rightarrow}-$ function for) the classical conditional when restricted to the subset $\{x, y\}$ of $\mathcal{V}$.

2. $f_{\rightarrow}$ satisfies Modus ponens, that is, for any $a, b \in \mathcal{V}$, if $a \rightarrow b \in D$ and $a \in D$, then $b \in D$.

3. For any $a, b \in \mathcal{V}, a \rightarrow b \in D$ if $a \leq b$.

Proposition 3.2 (Natural conditionals in 3-valued matrices) (a) Two designated values: Let $\mathcal{L}$ be a propositional language and $M$ be a 3-valued matrix where $\mathcal{V}$ is defined exactly as in $M K 3$ and $D=\{1,2\}$. Now, consider the $24 f_{\rightarrow}$ functions defined in the following general table:

$T I$

\begin{tabular}{l|lll}
$\rightarrow$ & 0 & 1 & 2 \\
\hline 0 & 2 & $a_{1}$ & 2 \\
1 & 0 & $a_{2}$ & $a_{3}$ \\
2 & 0 & $b_{1}$ & 2
\end{tabular}

where $a_{i}(1 \leq i \leq 3) \in\{1,2\}$ and $b_{1} \in\{0,1,2\}$. The set of functions contained in $T I$ is the set of all natural conditionals definable in $M$.

(b) One designated value: Now, let $M$ and $\mathcal{V}$ be as above but $D=\{2\}$. Consider the $6 f_{\rightarrow}$ functions in the following general table:

TII

\begin{tabular}{l|lll}
$\rightarrow$ & 0 & 1 & 2 \\
\hline 0 & 2 & 2 & 2 \\
1 & $a$ & 2 & 2 \\
2 & 0 & $b$ & 2
\end{tabular}

where $a \in\{0,1,2\}$ and $b \in\{0,1\}$. The set of functions contained in TII is the set of all natural conditionals definable in $M$.

Proof. It is obvious (cf. [14], [15]).

Next, the notion of a natural implicative 3-valued matrix is defined and all natural implicative expansions of MK3 are collected in Proposition 3.4.

Definition 3.3 (Natural implicative 3-valued matrices) Let $\mathcal{L}$ be a propositional language with the connective $\rightarrow$. And let $M$ be a 3-valued matrix where $\mathcal{V}$ and $D$ are defined as in Definition 2.6. Moreover, let $f_{\rightarrow}$ be one of the functions (defining one of the conditionals) in TI or TII (in Proposition 3.2). Then, it is said that $M$ is a natural implicative 3-valued matrix. (Notice that we are supposing that $\mathcal{V}$ is ordered as stated in Definition 2.6.)

Proposition 3.4 (Natural implicative expansions of MK3) (a) Two designated values: There are exactly 24 natural implicative expansions of MK3, 
Mt1, Mt2,..., Mt24, which are defined as follows. Each Mti $(1 \leq i \leq 24)$ is the structure $(\mathcal{V}, D, F)$ where $\mathcal{V}, D, f_{\wedge}, f_{\vee}$ and $f_{\neg}$ are defined exactly as in MK3 (cf. Definition 2.6, with $D=\{1,2\}$ ), whereas $f_{\rightarrow}$ is defined according to the table ti. Tables t1, t2, ..., t24 are displayed in Appendix 1; the notions of an Mti-interpretation, etc. are defined according to the general Definition 2.5. (b) Only one designated value: There are exactly 6 natural implicative expansions of MK3, Mt25, Mt26,..., Mt30, which are defined as follows. Each Mti $(25 \leq i \leq 30)$ is the structure $(\mathcal{V}, D, F)$ where $\mathcal{V}, D, f_{\wedge}, f_{\vee}$ and $f_{\neg}$ are defined exactly as in MK3 (cf. Definition 2.6, with $D=\{2\}$ ), whereas $f_{\rightarrow}$ is defined according to the table ti. Tables t25, t26, ..., t30 are displayed in Appendix 1; the notions of an Mti-interpretation, etc. are defined according to the general Definition 2.5.

Proof. Immediate by Proposition 3.2 and Definition 3.3.

However, there are only six implicative expansions of MK3 verifying all axioms and rules of Sylvan and Plumwood's minimal logic $B_{M}$. These six expansions are displayed in the following proposition.

Proposition 3.5 (The six natural implicative expansions of MK3) The logic $B_{M}$ is defined in Definition 4.4 below. The six natural implicative expansions of MK3 verifying all axioms and rules of Sylvan and Plumwood's $B_{M}$ are defined by adding to MK3 each one of the $f_{\rightarrow}$ functions in the truth tables below (designated values are starred).

\begin{tabular}{|c|c|c|c|c|c|c|c|c|c|c|c|c|c|c|}
\hline \multirow{4}{*}{$t 1$} & $\rightarrow$ & 0 & 1 & 2 & \multirow{4}{*}{$t^{2}$} & $\rightarrow$ & 0 & 1 & 2 & \multirow{4}{*}{ t3 } & $\rightarrow$ & 0 & 1 & 2 \\
\hline & $\overline{0}$ & $\overline{2}$ & 2 & 2 & & 0 & $\overline{2}$ & $\overline{2}$ & $\overline{2}$ & & 0 & $\overline{2}$ & $\overline{2}$ & $\overline{2}$ \\
\hline & 1 & 0 & 2 & 2 & & 1 & 1 & 2 & 2 & & 1 & 0 & 2 & 2 \\
\hline & $*_{2}$ & 0 & 0 & 2 & & $*_{2}$ & 0 & 1 & 2 & & $*_{2}$ & 0 & 1 & 2 \\
\hline & $\rightarrow$ & 0 & 1 & 2 & & $\rightarrow$ & 0 & 1 & 2 & & $\rightarrow$ & 0 & 1 & 2 \\
\hline & $\overline{0}$ & $\overline{2}$ & 2 & 2 & +5 & 0 & $\overline{2}$ & 2 & $\overline{2}$ & to & 0 & 2 & 2 & $\overline{2}$ \\
\hline & 1 & 1 & 2 & 2 & & ${ }^{*} 1$ & 0 & 1 & 2 & & ${ }^{* 1}$ & 0 & 2 & 2 \\
\hline & $*_{2}$ & 0 & 0 & 2 & & $*_{2}$ & 0 & 0 & 2 & & $*_{2}$ & 0 & 0 & 2 \\
\hline
\end{tabular}

Proof. Consider the lists of conditional tables in Appendix 1. (1) Two designated values: All tables except $\mathrm{t} 1, \mathrm{t} 4, \mathrm{t} 7, \mathrm{t} 10, \mathrm{t} 13, \mathrm{t} 16, \mathrm{t} 19$ and $\mathrm{t} 22$ falsify the rule Con. On the other hand, $\mathrm{t} 1, \mathrm{t} 4, \mathrm{t} 7$ and $\mathrm{t} 10$ falsify the rule Pref; and $\mathrm{t} 13$ and t19 falsify the rule Suf. Thus, we are left with t16 and t22 (i.e., t5 and t6 above). (2) Only one designated value: Tables t 25 and t26 falsify the rule Con. Thus, we are left with t27, t28, t29 and t30 (i.e., t1, t2, t 3 and t 4 above, respectively). (In case a tester is needed, the reader can use that in [9].)

The aim of this paper is to provide a reduced $\mathrm{RM}_{1}$-semantics for each one of the logics determined by these six implicative expansions of MK3. 


\section{The logics determined by the six natural im- plicative expansions of MK3 verifying all ax- ioms and rules of $B_{M}$}

In this section, we define the logics Lt1, Lt2,.., Lt6 determined by the natural implicative expansions of MK3, Mt1, Mt2,.., Mt6, verifying all axioms and rules of Sylvan and Plumwood's minimal logic $B_{M}$. We will generally refer by Lt $i$-logics to these six logics. We have tried to axiomatize the Lt $i$-logics with a common basis as wide as possible. In this sense, the six Lt $i$-logics are formulated as extensions of Routley and Meyer's disjunctive basic logic B, which is defined as shown in Definition 4.1 below (cf. [16]).

Definition 4.1 (The logic $\mathbf{B}^{\mathrm{d}}$ ) The logic $B^{d}$ is Routley and Meyer's disjunctive basic logic B. It is formulated as follows:

Axioms:

$$
\begin{aligned}
& \text { A1. }(A \wedge B) \rightarrow A /(A \wedge B) \rightarrow B \\
& \text { A2. }[(A \rightarrow B) \wedge(A \rightarrow C)] \rightarrow[A \rightarrow(B \wedge C)] \\
& \text { A3. } A \rightarrow(A \vee B) / B \rightarrow(A \vee B) \\
& \text { A4. }[(A \rightarrow C) \wedge(B \rightarrow C)] \rightarrow[(A \vee B) \rightarrow C] \\
& \text { A5. }[A \wedge(B \vee C)] \rightarrow[(A \wedge B) \vee(A \wedge C)] \\
& \text { A6. } A \rightarrow \neg \neg A \\
& \text { A\%. } \neg \neg A \rightarrow A
\end{aligned}
$$

Rules of inference:

$$
\begin{gathered}
\text { Adjunction (Adj): } A \& B \Rightarrow A \wedge B \\
\text { Modus Ponens (MP): } A \rightarrow B \& A \Rightarrow B \\
\text { Disjunctive Modus Ponens (dMP): } C \vee A \& C \vee(A \rightarrow B) \Rightarrow C \vee B \\
\text { Disjunctive Suffixing (dSuf): } D \vee(A \rightarrow B) \Rightarrow D \vee[(B \rightarrow C) \rightarrow(A \rightarrow C)] \\
\text { Disjunctive Prefixing (dPref): } D \vee(B \rightarrow C) \Rightarrow D \vee[(A \rightarrow B) \rightarrow(A \rightarrow C)] \\
\text { Disjunctive Contraposition (dCon): } C \vee(A \rightarrow B) \Rightarrow C \vee(\neg B \rightarrow \neg A)
\end{gathered}
$$

We note the following proposition.

Proposition 4.2 (Some theorems and rules of $\mathbf{B}^{\mathbf{d}}$ ) The following theorems and rules are provable in $B^{d}$.

$$
\begin{aligned}
& \text { T1. } A \rightarrow A \\
& \text { T2. } \neg(A \vee B) \leftrightarrow(\neg A \wedge \neg B) \\
& \text { T3. } \neg(A \wedge B) \leftrightarrow(\neg A \vee \neg B) \\
& \text { Suf. } A \rightarrow B \Rightarrow(B \rightarrow C) \rightarrow(A \rightarrow C) \\
& \text { Pref. } B \rightarrow C \Rightarrow(A \rightarrow B) \rightarrow(A \rightarrow C) \\
& \text { Con. } A \rightarrow B \Rightarrow \neg B \rightarrow \neg A
\end{aligned}
$$


Proof. Cf. [16], Chapter 4.

Next, we note how Routley and Meyer's B and Sylvan and Plumwood's $B_{M}$ are defined.

Definition 4.3 (Routley and Meyer's B) Routley and Meyer's basic logic $B$ is axiomatized with A1-A7, Adj, MP, Suf, Pref and Con (cf. Definition 4.1 and Proposition 4.2).

Definition 4.4 (Sylvan and Plumwood's $\mathrm{B}_{\mathrm{M}}$ ) Sylvan and Plumwood's minimal logic $B_{M}$ is axiomatized with A1-A5, Adj, MP, Suf, Pref, Con (cf. Definition 4.3) and, in addition, the axioms $A 6^{\prime},(\neg A \wedge \neg B) \rightarrow \neg(A \vee B)$ and $A 6^{\prime \prime}$, $\neg(A \wedge B) \rightarrow(\neg A \vee \neg B)$.

Thus, notice that $\mathrm{B}_{\mathrm{M}}$ is a sublogic of $\mathrm{B}$ (cf. [17]), but it is important to note that $\mathrm{B}$ and $\mathrm{B}^{\mathrm{d}}$ are different logics.

Proposition 4.5 ( $\mathbf{B}$ and $\mathbf{B}^{\mathrm{d}}$ are different logics) $B$ and $B^{d}$ are different logics, the former being included in the latter.

Proof. Consider the following set of truth-tables where 3 is the only designated valued:

\begin{tabular}{l|llll|l}
$\rightarrow$ & 0 & 1 & 2 & 3 & $\neg$ \\
\hline 0 & 3 & 3 & 3 & 3 & 3 \\
1 & 1 & 3 & 1 & 3 & 2 \\
2 & 1 & 1 & 3 & 3 & 1 \\
3 & 1 & 1 & 1 & 3 & 0
\end{tabular}

\begin{tabular}{l|llll}
$\wedge$ & 0 & 1 & 2 & 3 \\
\hline 0 & 0 & 0 & 0 & 0 \\
1 & 0 & 1 & 0 & 1 \\
2 & 0 & 0 & 2 & 2 \\
3 & 0 & 1 & 2 & 3
\end{tabular}

\begin{tabular}{l|llll}
$\vee$ & 0 & 1 & 2 & 3 \\
\hline 0 & 0 & 1 & 2 & 3 \\
1 & 1 & 1 & 3 & 3 \\
2 & 2 & 3 & 2 & 3 \\
3 & 3 & 3 & 3 & 3
\end{tabular}

These tables verify all axioms and rules of B but falsify the rule dMP $(C \vee$ $A \& C \vee(A \rightarrow B) \Rightarrow C \vee B)$.(Let $A, B$ and $C$ be the propositional variables $p_{i}, p_{k}$ and $p_{m}$, respectively, and consider any interpretation $I$ with $I\left(p_{i}\right)=$ $1, I\left(p_{k}\right)=0$ and $I\left(p_{m}\right)=2$ ). On the other hand, $\mathrm{B}$ is a sublogic of $\mathrm{B}^{\mathrm{d}}$, as shown in Proposition 4.2 and Definition 4.3.

In what follows, the Lti-logics are defined. In addition to A1-A7, the following list of axioms will be used.

$$
\begin{aligned}
& \text { A8. } A \rightarrow(A \rightarrow A) \\
& \text { A9. } \neg A \rightarrow[A \vee(A \rightarrow B)] \\
& \text { A10. } B \rightarrow[\neg B \vee(A \rightarrow B)] \\
& \text { A11. }[(A \rightarrow B) \wedge A] \rightarrow(\neg A \vee B) \\
& \text { A12. }[(A \rightarrow B) \wedge \neg B] \rightarrow(\neg A \vee B) \\
& \text { A13. }(A \vee \neg B) \vee(A \rightarrow B) \\
& \text { A14. }[(A \rightarrow B) \wedge(B \rightarrow C)] \rightarrow(A \rightarrow C) \\
& \text { A15. }[(A \rightarrow B) \wedge A] \rightarrow B \\
& \text { A16. }[(A \rightarrow B) \wedge \neg B] \rightarrow \neg A \\
& \text { A17. } A \vee \neg A
\end{aligned}
$$




$$
\begin{aligned}
& \text { A18. }(\neg A \wedge B) \rightarrow(A \rightarrow B) \\
& \text { A19. } A \rightarrow[B \vee \neg(A \rightarrow B)] \\
& \text { A20. } \neg B \rightarrow[\neg A \vee \neg(A \rightarrow B)] \\
& \text { A21. } \neg(A \rightarrow B) \rightarrow(A \vee \neg B) \\
& \text { A22. } \neg(A \rightarrow B) \rightarrow(A \wedge \neg B) \\
& \text { A23. } \neg(A \rightarrow B) \rightarrow \neg B \\
& \text { A24. } \neg(A \rightarrow B) \rightarrow A \\
& \text { A25. }(A \wedge \neg B) \rightarrow \neg(A \rightarrow B) \\
& \text { A26. }[\neg(A \rightarrow B) \wedge(\neg A \wedge B)] \rightarrow C
\end{aligned}
$$

Now, we begin by defining two basic extensions of $\mathrm{B}^{\mathrm{d}}, \mathrm{b}^{3}$ and $\mathrm{b}^{3} \mathrm{Ecq}$ (the label $\mathrm{b}^{3}$ is intended to abbreviate "basic logic contained in the six natural implicative expansions of $\mathrm{K} 3^{1}$ and $\mathrm{K} 3^{2}$ - cf. the last paragraph in section 2 - considered in this paper". The system $b^{3} E c q$ is the result of adding the rule Ecq (cf. Definition 4.7 below) to $\mathrm{b}^{3}$.

Definition 4.6 (The logic $\mathbf{b}^{3}$ ) The basic logic $b^{3}$ is the result of adding the following axioms to $B^{d}$ :

$$
\begin{gathered}
\text { A8. } A \rightarrow(A \rightarrow A) \\
A 9 . \neg A \rightarrow[A \vee(A \rightarrow B)] \\
A 10 . \quad B \rightarrow[\neg B \vee(A \rightarrow B)]
\end{gathered}
$$

Definition 4.7 (The logic $\mathbf{b}^{3} \mathbf{E c q}$ ) The basic logic $b^{3} E c q$ is the result of adding to $b^{3}$ the rule dEcq ("Disjunctive Ecq", where Ecq is "E contradictione quodlibet" - "Anything (follows) from a contradiction").

$$
d E c q: C \vee(A \wedge \neg A) \Rightarrow C \vee B
$$

We note some theorems provable in $\mathrm{b}^{3}$ and $\mathrm{b}^{3} \mathrm{Ecq}$.

Proposition 4.8 (Some theorems provable in $\mathbf{b}^{3}$ and $\mathbf{b}^{3} \mathbf{E c q}$ ) The following theorems are provable in $b^{3}$ and $b^{3} E c q$. In addition, the rule Ecq is provable in $b^{3} E c q$.

$$
\begin{aligned}
& \text { T4. } A \rightarrow[B \rightarrow(A \vee B)] \\
& \text { T5. }[\neg(A \rightarrow B) \wedge \neg A] \rightarrow A \\
& \text { T6. } A \rightarrow[\neg A \vee(\neg A \rightarrow B)] \\
& \text { T7. }(A \wedge \neg A) \rightarrow[B \vee[(A \wedge B) \rightarrow C]] \\
& \text { T8. }[\neg(A \rightarrow B) \wedge B] \rightarrow \neg B \\
& \text { Ecq. } A \wedge \neg A \Rightarrow B
\end{aligned}
$$


Proof. T4 is provable by $\mathrm{B}_{\mathrm{M}}$ and $\mathrm{A} 8$; $\mathrm{T} 5$ and $\mathrm{T} 6$, by $\mathrm{B}$ and $\mathrm{A} 9$; $\mathrm{T} 7$, by $\mathrm{B}$ and A9 in the form $\neg(A \wedge B) \rightarrow[(A \wedge B) \vee[(A \wedge B) \rightarrow C]]$; T8, by B and A10; finally, Ecq is immediate by dEcq.

Next, two more basic logics are defined $b_{1}^{3}$ and $b_{2}^{3}$. They are two mutually independent extensions of $\mathrm{b}^{3}$. The former is contained in Lt1, Lt2, Lt3 and Lt4; the latter, in Lt5 and Lt6.

Definition 4.9 (The logics $\mathbf{b}_{1}^{3}$ and $\mathbf{b}_{2}^{3}$ ) (a) The logic $b_{1}^{3}$ is axiomatized when adding the following axioms to $b^{3}$ Ecq:

$$
\begin{aligned}
& \text { A11. }[(A \rightarrow B) \wedge A] \rightarrow(\neg A \vee B) \\
& \text { A12. }[(A \rightarrow B) \wedge \neg B] \rightarrow(\neg A \vee B) \\
& \text { A13. }(A \vee \neg B) \vee(A \rightarrow B)
\end{aligned}
$$

(b) The logic $b_{2}^{3}$ is axiomatized when adding the following axioms to $b^{3}$ :

$$
\begin{aligned}
& \text { A14. }[(A \rightarrow B) \wedge(B \rightarrow C)] \rightarrow(A \rightarrow C) \\
& \text { A15. }[(A \rightarrow B) \wedge A] \rightarrow B \\
& \text { A16. }[(A \rightarrow B) \wedge \neg B] \rightarrow \neg A \\
& \text { A17. } A \vee \neg A \\
& \text { A18. }(\neg A \wedge B) \rightarrow(A \rightarrow B)
\end{aligned}
$$

We note some theorems of $b_{1}^{3}$ and $b_{2}^{3}$.

Proposition 4.10 (Some theorems of $\mathbf{b}_{1}^{3}$ and $\mathbf{b}_{2}^{3}$ ) The following are provable in $b_{2}^{3}$ :

$$
\begin{aligned}
& \text { T9. } A \rightarrow[B \vee \neg(A \rightarrow B)] \\
& \text { T10. } \neg B \rightarrow[\neg A \vee \neg(A \rightarrow B)] \\
& \text { T11. } A \vee(A \rightarrow B) \\
& \text { T12. } \neg B \vee(A \rightarrow B)
\end{aligned}
$$

On the other hand, T13 below is provable in $b_{1}^{3}$

$$
\text { T13. }(A \wedge \neg B) \rightarrow[\neg A \vee \neg(A \rightarrow B)]
$$

Proof. T9, T10 and T13 are derivable by B and A16, A15 and A11, respectively; then, T11 and T12 follow by $\mathrm{B}_{\mathrm{M}}$ and A17 together with A9 and A10, respectively.

Finally, the Lti-logics can be defined. (More conspicuous formulations of these logics can be found in Appendix 3.)

Definition 4.11 (The six implicative expansions of $\mathbf{K 3}^{1}\left(\mathrm{~K}^{2}\right)$ ) The six implicative expansions of $K 3^{1}$ or $K 3^{2}$, as the case may be, containing $B^{d}$ can be defined as follows. 
(a) Extensions of $b_{1}^{3}$ : The logics Lt1, Lt2, Lt3 and Lt4 are axiomatized by adding the following axioms to $b_{1}^{3}$ :

Lt1:

$$
\begin{aligned}
& \text { A19. } A \rightarrow[B \vee \neg(A \rightarrow B)] \\
& \text { A20. } \neg B \rightarrow[\neg A \vee \neg(A \rightarrow B)] \\
& \text { A21. } \neg(A \rightarrow B) \rightarrow(A \vee \neg B)
\end{aligned}
$$

Lt2:

$$
\text { A22. } \neg(A \rightarrow B) \rightarrow(A \wedge \neg B)
$$

\section{Lt3:}

$$
\begin{aligned}
& A 20 . \neg B \rightarrow[\neg A \vee \neg(A \rightarrow B)] \\
& \text { A23. } \neg(A \rightarrow B) \rightarrow \neg B
\end{aligned}
$$

\section{Lt4:}

$$
\begin{aligned}
& \text { A19. } A \rightarrow[B \vee \neg(A \rightarrow B)] \\
& \text { A24. } \neg(A \rightarrow B) \rightarrow A
\end{aligned}
$$

(b) Extensions of $b_{2}^{3}$ : The logics Lt5 and Lt6 are axiomatized by adding the following axioms to $b_{2}^{3}$ :

Lt5:

$$
\text { A25. }(A \wedge \neg B) \rightarrow \neg(A \rightarrow B)
$$

Lt6:

$$
\text { A26. }[\neg(A \rightarrow B) \wedge(\neg A \wedge B)] \rightarrow C
$$

Notice that $\mathrm{A} 21^{\prime},(\neg A \wedge B) \rightarrow(A \rightarrow B)$ (i.e., A18 of $\left.\mathrm{b}_{2}^{3}\right)$ is immediate in Lt1 by B and A21 (clearly, A22, A23 or A24 can be used instead of A21 to derive A21').

On the other hand, in Appendix 2, it is proved that the axiomatizations of Lt1, Lt2, Lt3, Lt4, Lt5 and Lt6 given in the present section are (deductively) equivalent (cf. Definition 2.3) to the original ones provided in [14] and [15].

Finally, the relations these logics maintain to each other are summarized in the following diagram, where the arrow is to be read as follows. Let $\mathrm{L}, \mathrm{L}^{\prime}$ be two logics in the diagram. $\mathrm{L} \rightarrow \mathrm{L}^{\prime}$ means that $\mathrm{L}$ is included in $\mathrm{L}^{\prime}$. More precisely, it means that, for any set of wffs $\Gamma$ and wff $A$, if $\Gamma \vdash_{\mathrm{L}} A$, then $\Gamma \vdash_{\mathrm{L}^{\prime}} A$. 


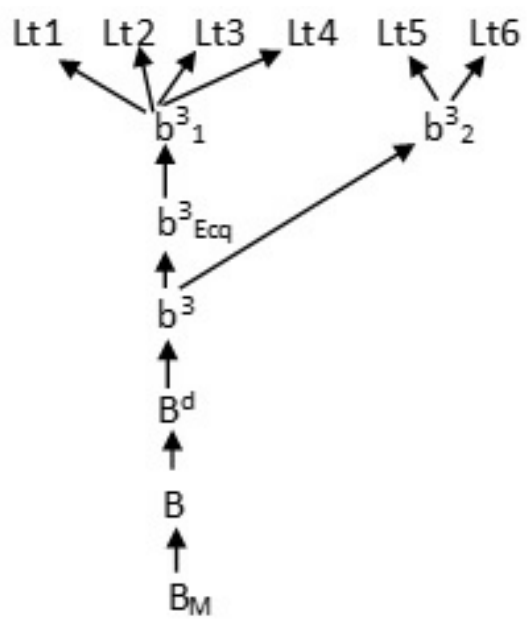

On the other hand, that there are not other relations between the logics in the diagram than those recorded (and, of course, those following from the transtivity of the relation expressed by $\rightarrow$ ) can be proved as follows (in case a tester is needed, the reader can use that in [9]; we can suppose that the soundness and completeness theorems in Sections 5 and 8 have been proved).

1. $\mathrm{b}^{3}$ is not included in $\mathrm{B}^{\mathrm{d}}$ : A9 and A10 are not theorems of relevant logic $\mathrm{R}$.

2. $\mathrm{b}_{\mathrm{Ecq}}^{3}$ is not included in $\mathrm{b}_{2}^{3}$ : Ecq is not validated in Mt5 and Mt6.

3. $b_{1}^{3}$ is not included in $b_{2}^{3}$ : Immediate by 2 .

4. $\mathrm{b}_{2}^{3}$ is not included in $\mathrm{b}_{1}^{3}: A \vee \neg A$ is not valid in Mt1, Mt2, Mt3 and Mt4.

5. Lt5 and Lt6 are not included in Lt1, Lt2, Lt3 and Lt4: Immediate by 4 .

6. Lt1, Lt2, Lt3 and Lt4 are not included in Lt4 and Lt5: Immediate by 2.

7. Lt5 and Lt6 are independent logics: $A \rightarrow[(A \rightarrow B) \rightarrow B]$ is not valid in Mt6; $B \rightarrow(A \rightarrow A)$ is not valid in Mt5.

8. Lt1, Lt2, Lt3 and Lt4 are independent logics: (a) $[(A \rightarrow B) \wedge \neg(A \rightarrow$ $B)] \rightarrow C$, valid in Mt1, is not valid in Mt2, Mt3 and Mt4. (b) $\neg(A \rightarrow$ $B) \rightarrow(A \wedge \neg B)$, valid in Mt2, is not valid in Mt1, Mt3 and Mt4. (c) $[A \wedge(A \rightarrow B)] \rightarrow B$, valid in Mt3, is not valid in Mt2 and Mt4; $A \rightarrow$ $(B \rightarrow A)$, valid in Mt3, is not valid in Mt1. (d) $[(A \rightarrow B) \wedge \neg B] \rightarrow \neg A$, valid in Mt4, is not valid in Mt2 and Mt3; $\neg A \rightarrow(A \rightarrow B)$, valid in Mt4, is not valid in Mt1. 


\section{Reduced Routley-Meyer semantics for the Lt $i$ - logics}

In this section a reduced Routley-Meyer semantics with a set of designated points (a reduced $\mathrm{RM}_{1}$-semantics) is provided for each one of the Lt $i$-logics. Given that the Lt $i$-logics are formulated as extensions of Routley and Meyer's disjunctive basic logic $B^{d}$, we begin by presenting a reduced $R_{1} M_{1}$-semantics for extensions of this last logic.

Definition 5.1 (EB ${ }^{\mathrm{d}}$-models) An $E B^{d}$-model, $M$, is a structure with at least the following items: (a) a set $K$ and an element of it, $O$; (b) a ternary relation $R$ and a unary operation $*$ defined on $K$ subject at least to the following definitions and postulates for all $a, b, c, d \in K$ :

$$
\begin{aligned}
& \text { d1. } a \leq b={ }_{d f} R O a b \\
& \text { d1'. } a=b=_{d f} a \leq b \quad \& \quad b \leq a \\
& \text { d2. } R^{2} a b c d={ }_{d f} \exists x \in K(\text { Rabx \& Rxcd }) \\
& \text { P1. } a \leq a \\
& \text { P2. }(a \leq b \& \text { Rbcd }) \Rightarrow \text { Racd } \\
& \text { P3. } a \leq b \Rightarrow b^{*} \leq a^{*} \\
& \text { P4. } a=a^{* *}
\end{aligned}
$$

(c) a valuation relation $\vDash$ from $K$ to the set of all formulas such that the following conditions (clauses) are satisfied for every propositional variable $p$, formulas $A, B$ and $a \in K$ :

$$
\begin{aligned}
& \text { (i). }(a \leq b \text { \& } a \vDash p) \Rightarrow b \vDash p \\
& \text { (ii). } a \vDash A \wedge B \text { iff } a \vDash A \& \text { \& } a \vDash B \\
& \text { (iii). } a \vDash A \vee B \text { iff } a \vDash A \text { or } a \vDash B \\
& \text { (iv). } a \vDash A \rightarrow B \text { iff for all } b, c \in K,(\text { Rabc \& } b \vDash A) \Rightarrow c \vDash B \\
& \text { (v). } a \vDash \neg A \text { iff } a^{*} \not \models A
\end{aligned}
$$

Additional elements of $M$ are (semantical) postulates $P j_{1}, \ldots, P j_{n}$.

Structures of the form $(O, K, R, *, \vDash)$ satisfying $d 1, d 1^{\prime}, d 2, P 1, P 2, P 3$, $P_{4}$ and clauses (i), (ii), (iii), (iv) and ( $v$ ) are the basic structures and in fact characterize the logic $B^{d}$ (they are labelled $B^{d}$-models). Introduction of additional postulates serve to determine extensions of $B^{d}$ interpretable in reduced $R M_{1}$-semantics.

Next, the notions of consequence and validity are defined. Then, we remark two lemmas which are useful in the soundness proofs to follow.

Definition $5.2\left(\mathbf{E B}^{\mathrm{d}}\right.$-consequence, $\mathbf{E B}^{\mathbf{d}}$-validity) Let a class of $E B^{d}$-models $\mathcal{M}$ be defined and $M \in \mathcal{M}$. For a non-empty set of formulas $\Gamma$ and formula $A$, 
$\Gamma \vDash_{M} A$ iff $O \vDash A$ if $O \vDash \Gamma(O \vDash \Gamma$ iff $\forall B \in \Gamma, O \vDash B)$. In particular, if $\Gamma=\emptyset$, $\vDash_{M} A$ ( $A$ is true in $\left.M\right)$ iff $O \vDash A$. Then, $\Gamma \vDash_{\mathcal{M}} A$ ( $A$ is $\mathcal{M}$-consequence of $\left.\Gamma\right)$ iff $\Gamma \vDash_{M} A$ for each $M \in \mathcal{M}$. In particular, $\vDash_{\mathcal{M}} A(A$ is valid in $\mathcal{M})$ iff $\vDash_{M} A$ for each $M \in \mathcal{M}$.

Lemma 5.3 (Hereditary Lemma) For any $E B^{d}$-model, $a, b \in K$ and formula $A,(a \leq b \quad \& \quad a \vDash A) \Rightarrow b \vDash A$.

Proof. Induction on the length of $A$. The conditional case is proved with P2 and the negation case with P3.

Lemma 5.4 (Entailment Lemma) Let a class of $E B^{d}$-models $\mathcal{M}$ be defined. For any formulas $A, B, \vDash_{\mathcal{M}} A \rightarrow B$ iff $(a \vDash A \Rightarrow a \vDash B$ for all $a \in K)$ in all $M$ $\in \mathcal{M}$.

Proof. From left to right $(\Rightarrow)$ by $\mathrm{P} 1$; from right to left $(\Leftarrow)$, by Lemma 5.3.

Let $\mathrm{L}$ be an $\mathrm{EB}^{\mathrm{d}}$-logic and L-models be defined. Below, it is proved that all theorems of $\mathrm{B}^{\mathrm{d}}$ are L-valid. Then, soundness of $\mathrm{B}^{\mathrm{d}}$ is a corollary of this fact.

Proposition 5.5 (All theorems of $\mathbf{B}^{\mathrm{d}}$ are $\mathbf{E B}^{\mathrm{d}}$-valid) For any formula A, if $\vdash_{B^{d}} A$, then $A$ is $E B^{d}$-valid (i.e., valid in any class of $E B^{d}$-models).

Proof. It can be found in [16], Chapter 4.

Corollary 5.6 (Soundness of $\mathbf{B}^{\mathbf{d}}$ ) For any wff $A$, if $\vdash_{B^{d}} A$, then $\vDash_{B^{d}} A$.

Proof. Immediate by Proposition 5.5, since a $\mathrm{B}^{\mathrm{d}}$-model is an $\mathrm{EB}^{\mathrm{d}}$-model.

In what follows, we proceed to the soundness proofs of the Lt $i$-logics. The basic notion is "corresponding postulate" (cf. [16], Chapter 4). We give a corresponding postulate to each one of the axioms A8 through A26 and the rule dEcq. Then, in order to prove soundness, these postulates are used as shown in Lemma 5.8. The section is ended with the proof of soundness of the Lt $i$-logics. Firstly, Lt $i$-models are defined. Then, (strong) soundness follows immediately from Definition 5.5 and Lemma 5.8.

Definition 5.7 (Postulates corresponding to A8-A26 and dEcq) Below, we provide postulates corresponding to each one of the axioms A8-A26 and the rule $d E c q$

$$
\begin{aligned}
& \text { PA8. } R a b c \Rightarrow(a \leq c \text { or } b \leq c) \\
& \text { PA9. } R a b c \Rightarrow\left(b \leq a \text { or } b \leq a^{*}\right) \\
& \text { PA10. } R a b c \Rightarrow\left(a \leq c \text { or } a^{*} \leq c\right) \\
& \text { PA11. Raaa or Raa* } a \\
& \text { PA12. Raa* } a^{*} \text { or } \operatorname{Raa}^{*} a \\
& P A 13 . R O a b \Rightarrow\left(O^{*} \leq b \text { or } a \leq O\right) \\
& P A 14 \text {. } R a b c \Rightarrow \exists x \in K(\operatorname{Rabx} \& \operatorname{Raxc}) \\
& \text { PA15. Raaa }
\end{aligned}
$$




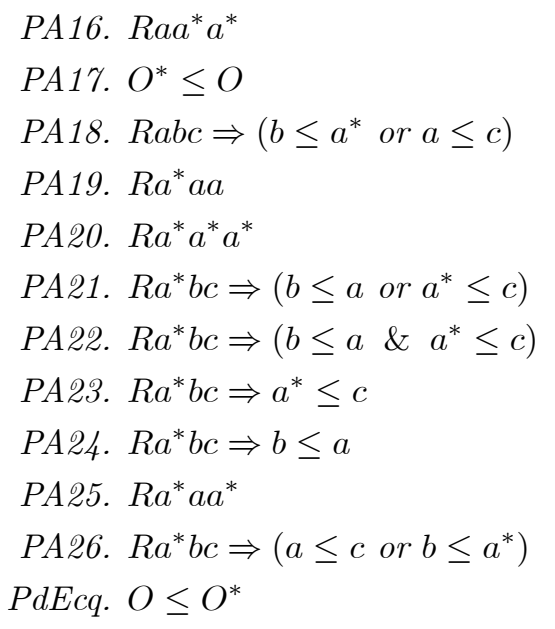

Lemma $5.8\left(\mathrm{~EB}^{\mathrm{d}}\right.$-validity of A8-A26 and dEcq) Let $\mathcal{M}$ be a class of $E B^{d}$ models and $M \in \mathcal{M}$. Then, for any $k(8 \leq k \leq 26), A k$ is true in $M$ if PAk holds in M. Moreover, $d E c q$ (disjunctive Ecq) preserves truth in $M$ if PdEcq holds in $M$.

Proof. The proof is similar to that given in [16], Chapter 4, for extensions of Routley and Meyer's basic logic B. So, it will suffice to prove some selected items (we lean upon the Entailment and Hereditary Lemmas, Lemma 5.3 and Lemma 5.4, respectively. By i, ii, etc., we refer to the clauses (i), (ii), etc., in Definition 5.1).

(a) A11, $[(A \rightarrow B) \wedge A] \rightarrow(\neg A \vee B)$, is true in $M$ : For reductio, suppose that there are wffs $A, B$ and $a \in K$ in M such that (1) $a \vDash(A \rightarrow B) \wedge A$ but (2) $a \not \models \neg A \vee B$. By 1 and ii, we have (3) $a \vDash A \rightarrow B$ and (4) $a \vDash A$; by 2, iii and v, we have (5) $a^{*} \vDash A$ and (6) $a \not \models B$; and by PA11, (7) Raaa or (8) Raa*a follows. Suppose 7 is the case, then we have (9) $a \vDash B$ (by $3,4,7$ and iv), contradicting 6 . On the other hand, suppose 8. Then, (10) $a \vDash B$ follows (by 3, 5, 8 and iv) contradicting again 6. Consequently, A11 is true in M.

(b) $A 13,(A \vee \neg B) \vee(A \rightarrow B)$, is true in $M$ : Suppose that there are wffs $A, B$ such that (1) $O \not \models(A \vee \neg B) \vee(A \rightarrow B)$. By iii and v, we have (2) $O \not \models A$, (3) $O^{*} \vDash B$ and (4) $O \not \models A \rightarrow B$; and by 4 and iv, there are $a, b \in K$ in $\mathrm{M}$ such that (5) ROab, (6) $a \vDash A$ and (7) $b \not \models B$. By PA13 and 5, either (8) $O^{*} \leq b$ or (9) $a \leq O$. Suppose 8 . Then, (10) $b \vDash B$ follows by 3 and 8 , contradicting 7 . On the other hand, suppose 9 . Then, we have (11) $O \vDash A$, by 6 and 9 , contradicting 2 .

(c) $A 17, A \vee \neg A$, is true in $M$ : Suppose that there is a wff $A$ such that (1) $O \not \models A$ or $(2) O^{*} \vDash A$. By PA17, (3) $O^{*} \leq O$, whence (4) $O \vDash A$ follows (by 2), contradicting 1.

(d) $A 26,[\neg(A \rightarrow B) \wedge(\neg A \wedge B)] \rightarrow C$, is true in $M$ : Suppose that there are wffs $A, B, C$ and $a \in K$ in M such that (1) $a^{*} \not \models A \rightarrow B$, (2) $a^{*} \not \models A$, (3) $a \vDash B$ and (4) $a \not \models C$. By 1 and iv, there are $b, c \in K$ in M such that (5) $R a^{*} b c$, (6) 
$b \vDash A$ and $(7) c \not \models B$. By PA26 and 5, either (8) $a \leq c$ or (9) $b \leq a^{*}$. But by 3 and $8,(10) c \vDash B$ follows, contradicting 7 ; and by 6 and 9 , we have (11) $a^{*} \vDash A$, which contradicts 2 .

(e) $d E c q$, if $C \vee(A \wedge \neg A)$, then $C \vee B$, preserves truth in $M$ : Suppose that there are wffs $A, B, C$ such that (1) $O \vDash C \vee(A \wedge \neg A)$ but (2) $O \not \models C \vee B$. By 2 and iii, we have (3) $O \not \models C$ and (4) $O \not \models B$; and by 1, 3 and iii, we get (5) $O \vDash A \wedge \neg A$, whence (6) $O \vDash A$ and (7) $O \vDash \neg A$ follow by ii. But, by 7 and v, we have (8) $O^{*} \not \models A$, which contradicts (9) $O^{*} \vDash A$, immediate by $\operatorname{PdEcq}\left(O \leq O^{*}\right)$ and 6 .

Finally, once the notion of an Lti-model defined, we prove soundness of the Lt $i$-logics.

Definition 5.9 (Lt $i$-models) An Lti-model is defined when adding to $B^{d}$-models the semantical postulates corresponding to the axioms added to $B^{d}$ for axiomatizing Lti. For example, Lt3-models are structures $(O, K, R, *, \models)$ where $O, K$, $R, *$ and $\vDash$ are defined exactly as in Definition 5.1, save for the addition of the postulates PA8, PA9, PA10, PA11, PA12, PA13, PA20 and PA23. (The notions of Lt3-consequence and Lt3-validity are defined according to the general Definition 5.2).

Theorem 5.10 (Soundness of the Lt $i$-logics) For any $i(1 \leq i \leq 6)$, set of formulas $\Gamma$ and formula $A$, if $\Gamma \vdash_{L t i} A$, then $\Gamma \vDash_{L t i} A$.

Proof. By Proposition 5.5 and Lemma 5.8, given Definition 5.9.

\section{Extension and primeness lemmas}

In this section, the extension and primeness lemmas are remarked (cf. [16]; cf. also [6]). These lemmas are essentially used in the completeness proofs in section 8 .

Definition 6.1 (EB ${ }^{\mathbf{d}}$-theories) Let $L$ be an $E B^{d}$-logic. An L-theory is a set of formulas closed under Adjunction (Adj) and L-entailment (L-ent). That is, $a$ is an L-theory if whenever $A, B \in a$, then $A \wedge B \in a$; and if whenever $A \rightarrow B$ is a theorem of $L$ and $A \in a$, then $B \in a$.

By the term $\mathrm{EB}^{\mathrm{d}}$-theory, we will generally refer to any theory defined upon an $\mathrm{EB}^{\mathrm{d}}$-logic as just indicated. The classes of $\mathrm{EB}^{\mathrm{d}}$-theories of interest in the present paper are remarked in the following definition.

Definition 6.2 (Classes of $\mathbf{E B}^{\mathrm{d}}$-theories) Let $L$ be an $E B^{d}$-logic and $a$ an L-theory. We set:

1. a is prime iff whenever $A \vee B \in a$, then $A \in a$ or $B \in a$.

2. a is empty iff it contains no formulas. 
3. $a$ is regular iff a contains all theorems of $L$.

4. $a$ is trivial iff every formula belongs to it.

5. a is a-consistent (consistent in an absolute sense) iff a is not trivial.

6. $a$ is n-consistent (consistent according to the standard concept of consistency) iff $A \wedge \neg A \notin$ a for any formula $A$.

Definition 6.3 (Disjunctive $\mathbf{E B}^{\mathrm{d}}$-derivability) Let $L$ be an $E B^{d}$-logic and $\Gamma, \Theta$ sets of formulas. $\Theta$ is disjunctively derivable from $\Gamma$ in $L$ (in symbols, $\left.\Gamma \vdash_{L}^{d} \Theta\right)$ iff $A_{1} \wedge \ldots \wedge A_{n} \vdash_{L} B_{1} \vee \ldots \vee B_{m}$ for some formulas $A_{1}, \ldots, A_{n} \in \Gamma$ and $B_{1}, \ldots, B_{n} \in \Theta$.

Definition $6.4\left(\mathrm{~EB}^{\mathrm{d}}\right.$-maximal sets) Let $L$ be and $E B^{d}$-logic. $\Gamma$ is an $L$ maximal set of formulas iff $\Gamma \nvdash_{L}^{d} \bar{\Gamma}(\bar{\Gamma}$ is the complement of $\Gamma)$.

Once the notions of Disjunctive $\mathrm{EB}^{\mathrm{d}}$-derivability and $\mathrm{EB}^{\mathrm{d}}$-maximal set being defined, the Extensions to maximal sets and Primeness Lemmas are proved by leaning on the Preliminary Lemma to the Extension Lemma.

Lemma 6.5 (Preliminary Lemma to the Extension Lemma) Let $L$ be an $E B^{d}$-logic with no other primitive rules than Adjunction, Modus Ponens, disjunctive Modus Ponens, disjunctive Suffixing, disjunctive Prefixing and disjunctive Contraposition or just with no other primitive rules than these ones and the rule disjunctive Ecq. Then, for formulas $A, B_{1}, \ldots, B_{n}$, if $\left\{B_{1}, \ldots, B_{n}\right\} \vdash_{L} A$, then for any formula $C, C \vee\left(B_{1} \wedge \ldots \wedge B_{n}\right) \vdash_{L} C \vee A$.

Proof. Cf. [6], p. 27 or Lemma 7.3 in [13].

Lemma 6.6 (Extensions to maximal sets) Let $L$ be an $E B^{d}$-logic with no other primitive rules than Adjunction, Modus Ponens, disjunctive Modus Ponens, disjunctive Suffixing, disjunctive Prefixing and disjunctive Contraposition or just with no other primitive rules than these ones and the rule disjunctive Ecq. For sets $\Gamma, \Theta$ of formulas such that $\Gamma \nvdash_{L}^{d} \Theta$, there are sets of formulas $\Gamma^{\prime}$, $\Theta^{\prime}$ such that $\Gamma \subseteq \Gamma^{\prime}, \Theta \subseteq \Theta^{\prime}, \Theta^{\prime}=\bar{\Gamma}^{\prime}$ and $\Gamma^{\prime} \nvdash_{L}^{d} \Theta^{\prime}$ (that is, $\Gamma^{\prime}$ is an L-maximal set such that $\left.\Gamma^{\prime} \nvdash_{L}^{d} \Theta^{\prime}\right)$.

Proof. Cf. Lemma 9 in [6] or Chapter 4 in [16], or Lemma 7.4 in [13].

Lemma 6.7 (Primeness) Let $L$ be an $E B^{d}$-logic with no other primitive rules than Adjunction, Modus Ponens, disjunctive Modus Ponens, disjunctive Suffixing, disjunctive Prefixing and disjunctive Contraposition or just with no other primitive rules than these ones and the rule disjunctive Ecq. If $\Gamma$ is an $L$ maximal set, then it is a prime L-theory closed under the rules of $L$.

Proof. Cf. Lemma 8 in [6]. 


\section{Preliminaries to the completeness theorems}

In this section, we prove a series of preliminary lemmas to be used in the completeness proofs of the Lti-logics. As in section 6, we essentially follow the terminology and strategy of [16], Chapter 4 . Firstly, we define the notions of a fundamental $\mathrm{EB}^{\mathrm{d}}$-theory and that of a $\mathcal{T}$-theory.

Definition 7.1 (Fundamental theories) Let L be an $E B^{d}$-logic. An L-theory is fundamental if it is a regular and prime L-theory closed under the primitive rules of derivation of $L$.

By the term fundamental $\mathrm{EB}^{\mathrm{d}}$-theory (or simply, fundamental theory), we will generally refer to any theory defined upon an $\mathrm{EB}^{\mathrm{d}}$-logic as shown in Definition 7.1.

Definition 7.2 ( $\mathcal{T}$-theories) Let $\mathcal{T}$ be a fundamental theory. $A \mathcal{T}$-theory is a set of formulas closed under Adjunction (Adj) and $\mathcal{T}$-entailment $(\mathcal{T}$-ent). That is, $a$ is a $\mathcal{T}$-theory if whenever $A, B \in a$, then $A \wedge B \in a$; if whenever $A \rightarrow B \in \mathcal{T}$ and $A \in a$, then $B \in a$.

It is obvious that $\mathcal{T}$-theories are $\mathrm{EB}^{\mathrm{d}}$-theories.

The main notions needed in order to define the canonical model are recorded in the following definition. These notions are used for defining canonical models in section 8 (cf. Definition 8.3).

Definition 7.3 (Main notions for defining canonical models) Let $\mathcal{T}$ be a fundamental theory and $K^{T}$ be the set of all $\mathcal{T}$-theories. Then, the ternary relation $R^{T}$ is defined in $K^{T}$ as follows: for all formulas $A, B$ and $a, b, c \in K^{T}$, $R^{T}$ abc iff $(A \rightarrow B \in a \& A \in b) \Rightarrow B \in c$. Next, let $K^{P}$ be the set of all prime $\mathcal{T}$-theories and $R^{P}$ the restriction of $R^{T}$ to $K^{P}$. On the other hand, let $K^{C}$ be the set of all non-empty, a-consistent prime $\mathcal{T}$-theories and $*^{C}$ be defined on $K^{C}$ as follows: for all $a \in K^{C}, a^{* C}=\{A \mid \neg A \notin a\}$. Finally, the relation $\vDash^{C}$ is defined as follows: for each formula $A$ and $a \in K^{C}, a \vDash^{C} A$ iff $A \in a$.

In the rest of this section, we prove a series of lemmas which will be used in the completeness proofs in section 8. These lemmas are proved for $\mathrm{Eb}^{3}$-logics (the logic $\mathrm{B}^{\mathrm{d}}$ is not sufficiently strong). Thus, we suppose that we are given a fundamental $\mathrm{Eb}^{3}$-theory $\mathcal{T}$ upon which the items $K^{T}, K^{C}, R^{C},{ }^{C}$ and $\vDash^{C}$ are defined as shown in Definition 7.3.

Lemma 7.4 (Defining $x$ for $a, b$ in $R^{T}$ ) Let $a, b$ be non-empty $\mathcal{T}$-theories. The set $x=\{B \mid \exists A(A \rightarrow B \in a \& A \in b)\}$ is a non-empty $\mathcal{T}$-theory such that $R^{T} a b x$.

Proof. It is easy to show that $x$ is a $\mathcal{T}$-theory (use dSuf to prove that $x$ is closed under $\mathcal{T}$-ent). Next, $R^{T} a b x$ is immediate by definition of $R^{T}$. Finally, $x$ is non-empty: let $A \in a, B \in b$. By T4 and $R^{T} a b x, A \vee B \in x$. 
Lemma 7.5 (Extending $b$ in $R^{T} a b c$ to a member in $K^{C}$ ) Let $b$ be a nonempty $\mathcal{T}$-theory, a a non-empty and a-consistent $\mathcal{T}$-theory and $c$ an a-consistent $\mathcal{T}$-theory such that $R^{T} a b c$. Then, there is an a-consistent (and non-empty) prime $\mathcal{T}$-theory $x$ such that $b \subseteq x$ and $R^{T}$ axc.

Proof. By using the Extension Lemma or Zorn's Lemma, $b$ is extended to a prime theory $x$ such that $b \subseteq x$ and $R^{T}$ axc (cf. [17], pp. 309, ff.). Next, it is shown that $x$ is a-consistent. Suppose it is not. (We use Proposition 4.8.) Let $A \in a$ and $B$ be a wff belonging to neither $a$ nor $c$. By T6, $A \rightarrow[\neg A \vee(\neg A \rightarrow B)]$. So, $\neg A \vee(\neg A \rightarrow B) \in a$, whence by primeness of $a$, either (1) $\neg A \in a$ or (2) $\neg A \rightarrow B \in a$. Let us consider case 2. As $x$ is supposed to be trivial, $\neg A \in x$. But then $B \in c\left(R^{T} a x c, \neg A \rightarrow B \in a, \neg A \in x\right.$ and definition of $R^{T}$ ) contradicting our hypothesis. Let us now examine case 1. Firstly, notice that $A \wedge \neg A \in a$. Next, we have for arbitrary $C, B \vee[(A \wedge B) \rightarrow C] \in a$ by applying T7 $((A \wedge \neg A) \rightarrow[B \vee[(A \wedge B) \rightarrow C]])$, whence $(A \wedge B) \rightarrow C \in a$ since $B \notin a$. Now, $A \wedge B \in x$ ( $x$ is supposed to be a-inconsistent). Thus, $C \in c$ $\left((A \wedge B) \rightarrow C \in a, A \wedge B \in x, R^{T} a x c\right)$, contradicting the a-consistency of $c$.

Lemma 7.6 (Extending $a$ in $R^{T} a b c$ to a member in $K^{C}$ ) Let $a, b$ be nonempty $\mathcal{T}$-theories and $c$ be an a-consistent, prime $\mathcal{T}$-theory such that $R^{T}$ abc. Then, there is an a-consistent (and non-empty) prime $\mathcal{T}$-theory $x$ such that $a \subseteq x$ and $R^{T} x b c$.

Proof. As in the previous lemma, it is shown that there is a prime theory $x$ such that $a \subseteq x$ and $R^{T} x b c$. Next, it is shown that $x$ is a-consistent. Suppose it is not and let $A \in b$ and $B$ be an arbitrary wff. As $x$ is supposed to be trivial, $A \rightarrow B \in x$. Then, $B \in c\left(R^{T} x b c, A \rightarrow B \in x, A \in b\right.$ and definition of $\left.R^{T}\right)$ contradicting the a-consistency of $c$.

Consider now the following definition.

Definition 7.7 (The relation $\leq^{C}$ ) For any $a, b \in K^{C}, a \leq^{C} b$ iff $R^{C} \mathcal{T} a b$.

The following lemma shows that the relation $\leq^{C}$ is just set inclusion between a-consistent and non-empty prime $\mathcal{T}$-theories.

Lemma $7.8\left(\leq^{C}\right.$ and $\subseteq$ are coextensive) For any $a, b \in K^{C}, a \leq^{C} b$ iff $a \subseteq b$.

Proof. From left to right, it is immediate by using T1 of $\mathrm{B}^{\mathrm{d}}$. Suppose now $a \subseteq b$ for $a, b \in K^{C}$. Clearly $R^{C} \mathcal{T} a a$ (cf. Definitions 6.1 and 7.2). By the hypothesis, $R^{C} \mathcal{T} a b$, i.e., $a \leq^{C} b$ by Definition 7.7.

Lemma 7.9 (Extension to prime $\mathcal{T}$-theories) Let a be a $\mathcal{T}$-theory and $A$ a wff such that $A \notin a$. Then, there is a prime $\mathcal{T}$-theory $x$ such that $a \subseteq x$ and $A \notin x$. 
Proof. By direct application of Zorn's Lemma as in [16], Chapter 4, pp. 310311.

In what follows, we investigate the operation $*^{C}$.

Lemma 7.10 (Primeness of $*$-images) Let $a$ be a prime $\mathcal{T}$-theory. Then, (1) $a^{*^{C}}$ is a prime $\mathcal{T}$-theory as well; (2) for any wff $A, \neg A \in a^{*^{C}}$ iff $A \notin a$.

Proof. As there is no danger of confusion between $a^{*}$ in $K$ and the canonical L-theory $a^{*^{C}}$ in $K^{C}$, we omit the supersript ${ }^{C}$ above $*$ in this and the proofs to follow. (1) $a^{*}$ is closed under $\mathcal{T}$-ent by Con; $a^{*}$ is closed under Adj by T3; $a^{*}$ is prime by T2. (2) By A6 and A7.

Lemma $7.11\left(*^{C}\right.$ is an operation on $\left.K^{C}\right)$ Let a be an a-consistent and nonempty prime $\mathcal{T}$-theory. Then, $a^{*}$ is an a-consistent and non-empty prime $\mathcal{T}$ theory as well.

Proof. By Lemma 7.10, $a^{*}$ is a prime $\mathcal{T}$-theory. Next, it is shown that if $a$ is a-consistent and non-empty, then $a^{*}$ is also a-consistent and non-empty. (1) $a^{*}$ is a-consistent. As $a$ is non-empty, there is some wff $A$ such that $A \in a$. Then, $\neg A \notin a^{*}$, by Lemma 7.10(2). (2) $a^{*}$ is non-empty. As $a$ is a-consistent, there is some wff $A$ such that $A \notin a$. Then, $\neg A \in a^{*}$ by Lemma 7.10(2).

Finally, it is proved that the relation $\vDash^{C}$ obeys requirements (clauses) (i)-(v) in the definition of an $\mathrm{EB}^{\mathrm{d}}$-model (cf. Definition 5.1).

Lemma $7.12\left(\vDash^{C}\right.$ and clauses (i)-(v)) For any $a, b, c \in K^{C}$ and wffs $A, B$,

(i). $\left(a \leq^{C} b \& a \vDash^{C} p\right) \Rightarrow b \vDash^{C} p$

(ii). $a \vDash^{C} A \wedge B$ iff $a \vDash^{C} A$ and $a \vDash^{C} B$

(iii). $a \vDash^{C} A \vee B$ iff $a \vDash^{C} A$ or $a \vDash^{C} B$

(iv). $a \vDash^{C} A \rightarrow B$ iff for all $b, c \in K^{C},\left(R^{C}\right.$ abc and $\left.b \vDash^{C} A\right) \Rightarrow c \vDash^{C} B$

(v). $a \vDash^{C} \neg A$ iff $a^{*^{C}} \not \nvdash^{C} A$

Proof. (i) is immediate by Lemma 7.8; (ii) follows by A1 and closure of $a$ under Adj; (iii) is proved by A3 and primeness of $a$; and (v) and (iv) (from left to right) are immediate by Definition 7.3. So, let us prove (iv) from right to left. For wffs $A, B$ and $a \in K^{C}$, suppose $A \rightarrow B \notin a$ (i.e., $a \not \nvdash^{C} A \rightarrow B$ ). We prove that there are $x, y \in K^{C}$ such that $R^{C} a x y, A \in x$ (i.e., $x \vDash^{C} A$ ) and $B \notin y$ (i.e., $y \not \nvdash^{C} B$ ). Consider the sets $z=\{C \mid A \rightarrow C \in \mathcal{T}\}$ and $u=\{C \mid \exists D(D \rightarrow C \in a \quad \& \quad D \in z)\}$. Firstly, notice that $A \in z$, since $A \rightarrow A \in \mathcal{T}$ by $\mathrm{T} 1$ of $\mathrm{B}^{\mathrm{d}}$. Then, $z$ and $u$ are easily shown $\mathcal{T}$-theories such that $R^{T} a z u$. Now, $B \notin u$ (if $B \in u$, then $A \rightarrow B \in a$ contradicting the hypothesis). Moreover, $u$ is not empty by Lemma 7.4. Then, by Lemma 7.9, there is a (a-consistent and non-empty) prime $\mathcal{T}$-theory $y$ such that $u \subseteq y$ and $B \notin y$. Clearly, $R^{T} a z y$ (cf. Definition 7.3). Next, by using Lemma 7.5, $z$ is extended to an a-consistent, non-empty and prime $\mathcal{T}$-theory $x$ such that $z \subseteq x$ 
and $R^{C}$ axy. Clearly, $A \in x$. Therefore, we have a-consistent and non-empty prime $\mathcal{T}$-theories $x, y$ such that $A \in x, B \notin y$ and $R^{C} a x y$, as was to be proved.

\section{Completeness of the Lt $i$-logics}

Definition 8.1 (The set of consequences of $\Gamma$ in $\mathbf{L}$ ) Let $L$ be an $E B^{d}$-logic. The set of consequences in $L$ of a set of formulas $\Gamma$ (in symbols $C n \Gamma[L]$ ) is defined as follows: $C n \Gamma[L]=\left\{A \mid \Gamma \vdash_{L} A\right\}$.

It is obvious that $\mathrm{Cn} \Gamma[\mathrm{L}]$ is a regular L-theory.

Now we have:

Proposition 8.2 (The building of $\mathcal{T}$ ) Let $L$ be an $E B^{d}$-logic with no other primitive rules than Adjunction, Modus Ponens, disjunctive Modus Ponens, disjunctive Suffixing, disjunctive Prefixing and disjunctive Contraposition or just with no other primitive rules than these ones and the rule disjunctive Ecq, $\Gamma$ a set of wffs and $A$ a wff such that $\Gamma \nvdash_{L} A$. Then, there is a fundamental L-theory $\mathcal{T}$ such that $\Gamma \subseteq \mathcal{T}$ and $A \notin \mathcal{T}$.

Proof. Assuming the hypothesis of Proposition 8.2, suppose $\Gamma \nvdash_{\mathrm{L}} A$. Then, $A \notin \mathrm{Cn} \Gamma[\mathrm{L}]$ and so $\mathrm{Cn} \Gamma[\mathrm{L}] \nvdash_{\mathrm{L}}^{\mathrm{d}}\{A\}$ : otherwise $\left(B_{1} \wedge \ldots \wedge B_{n}\right) \vdash_{\mathrm{L}} A$ for some $B_{1}, \ldots, B_{n} \in \Gamma$ whence $A$ would be in $\operatorname{Cn} \Gamma[\mathrm{L}]$. Next, Lemmas 6.6 and 6.7 apply and there is some fundamental L-theory $\mathcal{T}$ such that $\Gamma \subseteq \mathcal{T}$ (since $\Gamma \subseteq \mathrm{Cn} \Gamma[\mathrm{L}]$ ) and $A \notin \mathcal{T}$. (Notice that $\mathcal{T}$ is a-consistent in addition to being fundamental.)

Leaning on this theory $\mathcal{T}$, the canonical L-model is defined and $\Gamma \nvdash_{\mathrm{L}} A$ is proved.

Definition 8.3 (Canonical EB ${ }^{\mathrm{d}}$-models) Let $\mathcal{T}, K^{C}, R^{C}, *^{C}$ and $\vDash^{C}$ be defined upon the fundamental $L$-theory $\mathcal{T}$ as shown in Definition 7.3. Then, the structure $\left(\mathcal{T}, K^{C}, R^{C}, *^{C}, \models^{C}\right)$ is a canonical L-model.

Proposition 8.4 (The postulates are canonically valid) Let $L$ be an Ltilogic. Then, (1) P1, P2, P3 and P4 hold in all canonical L-models. (2) PAk holds in the canonical $L$-model if $A k$ is provable in $L(8 \leq k \leq 26)$. (PdEcq holds in the canonical L-model if $d E c q$ is provable in L.)

Proof. The proof is similar to that provided in [16], Chapter 4, for extensions of Routley and Meyer's basic logic B. We prove Proposition 8.4 for the postulates used above in Lemma 5.8 .

(a) PA11, Raaa or Raa*a, is provable in the canonical Lti-model: Let $a \in$ $K^{C}$ and suppose, for reductio, (1) not- $R^{C} a a a$ and (2) not- $R^{C} a a^{*} a$. By 1 and Definition 7.3, we have, for wffs $A, B$, (3) $A \rightarrow B \in a$, (4) $A \in a$ and (5) $B \notin a$; by 2 and Definition 7.3, we get, for wffs $C, D,(6) C \rightarrow D \in a,(7) C \in a^{*}$ and (8) $D \notin a$. By 3, 6, B and Definition 7.3, we have (9) $(A \vee C) \rightarrow(B \vee D) \in a$; 
and by 4 and 7 , we get (10) $A \vee C \in a$ and (11) $A \vee C \in a^{*}$. Consider now A11 in the form (12) $\{[(A \vee C) \rightarrow(B \vee D)] \wedge(A \vee C)\} \rightarrow[\neg(A \vee C) \vee(B \vee D)]$. By 9,10 and 12, we obtain $(13) \neg(A \vee C) \vee(B \vee D) \in a$. Now, (14) $\neg(A \vee C) \notin a$ is derivable from 11 by Definition 7.3, whence (15) $B \vee D \in a$ follows by 13 and primeness of $a$. But 5 and 8 contradict 15 .

(b) $P A 13, R O a b \Rightarrow\left(O^{*} \leq b\right.$ or $\left.a \leq O\right)$, is provable in the canonical Ltimodel: Let $a, b \in K^{C}$ and suppose (1) $R^{C} \mathcal{T} a b$, i.e., $a \leq^{C} b$ (cf. Lemma 7.8). Moreover, for reductio, suppose (2) $\mathcal{T}^{*} \not^{C} b$ and (3) $a \not^{C} \mathcal{T}$. By 2,3 and Lemma 7.8, we have for wffs $A, B$, (4) $A \in \mathcal{T}^{*}$, (5) $A \notin b$, (6) $B \in a$ and (7) $B \notin \mathcal{T}$. By A13 and regularity of $\mathcal{T}$, we have $(8)(B \vee \neg A) \vee(B \rightarrow A) \in \mathcal{T}$. Now, (9) $\neg A \notin \mathcal{T}$ is derivable from 4 and Definition 7.3. So, we get (10) $B \rightarrow A \in \mathcal{T}$, by $7,8,9$ and primeness of $\mathcal{T}$. Finally, (11) $A \in b$ is derivable by 1,6 and 10 (cf. Definition 7.3). But 5 and 11 contradict each other.

(c) PA17, $O^{*} \leq O$, is provable in the canonical Lti-model: We prove $\mathcal{T}^{*} \subseteq \mathcal{T}$, whence $\mathcal{T}^{*} \leq^{C} \mathcal{T}$ follows by Lemma 7.8. Suppose for a given formula $A$, (1) $A \in \mathcal{T}^{*}$. Then, (2) $\neg A \notin \mathcal{T}$ by Definition 7.3. By A17 and regularity and primeness of $\mathcal{T}$, we have (3) $A \in \mathcal{T}$ or $\neg A \in \mathcal{T}$. Consequently, (4) $A \in \mathcal{T}$, that is, (5) $\mathcal{T}^{*} \subseteq \mathcal{T}$.

(d) PA26, $R a^{*} b c \Rightarrow\left(a \leq c\right.$ or $\left.b \leq a^{*}\right)$, is provable in the canonical Ltimodel: Suppose that there are $a, b, c \in K^{C}$ such that (1) $R a^{*} b c$ but (2) $a \Varangle^{C} c$ and (3) $b \Varangle^{C} a^{*}$. Then, there are wffs $A, B$ such that (4) $A \in a$, (5) $A \notin c$, (6) $B \in b$ and (7) $B \notin a^{*}$, i.e., $\neg B \in a$. By 1, 5, 6 and Definition 7.3, we have $(8) B \rightarrow A \notin a^{*}$, i.e., $\neg(B \rightarrow A) \in a$. Now, we use A26 in the form (9) $[\neg(B \rightarrow A) \wedge(\neg B \wedge A)] \rightarrow C(C$ is an arbitrary formula $)$. By $4,7,8$ and 9 , we get (10) $C \in a$, contradicting the a-consistency of $a$.

(e) PdEcq, $O \leq O *$, is provable in the canonical Lti-model: We prove $\mathcal{T} \subseteq$ $\mathcal{T}^{*}$, whence $\mathcal{T} \leq{ }^{C} \mathcal{T}^{*}$ follows by Lemma 7.8. Suppose then that there is some wff $A$ such that (1) $A \in \mathcal{T}$ but (2) $A \notin \mathcal{T}^{*}$. Then (3) $\neg A \in \mathcal{T}$. But by Ecq, 1 and $3 B \in \mathcal{T}$ follows for arbitrary $B$, contradicting the a-consistency of $\mathcal{T}$.

Proposition 8.5 (The canonical L-model is an L-model) Let $L$ be an Ltimodel. The canonical L-model is indeed an L-model.

Proof. Given Definition 8.3 and Proposition 8.2, the proof follows by Lemma $7.11\left(*^{C}\right.$ is an operation on $K^{C}$ ), Lemma 7.12 (Adequacy of the canonical clauses) and Lemma 8.4 (The postulates hold canonically).

Finally, we prove completeness.

Theorem 8.6 (Completeness of Lt $i$-logics) Let Lti be any of the logics Lt1Lt6. For any set of formulas $\Gamma$ and a formula $A$, if $\Gamma \vDash_{L t i} A$, then $\Gamma \vdash_{L t i} A$.

Proof. For some set of formulas $\Gamma$ and formula $A$ suppose $\Gamma \nvdash_{\mathrm{Lt} i} A$. We prove $\Gamma \nvdash_{\mathrm{Lt} i} A$. If $\Gamma \nvdash_{\mathrm{Lt} i} A$, then by Proposition 8.2 , there is a regular and a-consistent prime Lt $i$-theory $\mathcal{T}$ closed under the primitive rules of Lt $i$ such that $\Gamma \subseteq \mathcal{T}$ and $A \notin \mathcal{T}$. Then, the canonical Lt $i$-model is defined upon $\mathcal{T}$ as indicated in Definition 8.3. By Proposition 8.5, the canonical Lt $i$-model is an 
Lti-model. Then, $\Gamma \not \nvdash^{C} A$ since $\mathcal{T} \vDash^{C} \Gamma$ but $\mathcal{T} \not \nvdash^{C} A$, whence, by Definition 5.2, $\Gamma \nvdash_{\mathrm{Lt} i} A$ follows, as it was to be proved.

If $\Gamma$ is the empty set, let Lt $i$ be the set of all theorems of Lt $i$. Then, Lt $i \nvdash_{\mathrm{Lt} i} A$ and we proceed similarly as above.

\section{A Appendix 1. List of the conditional tables in Proposition 3.4}

We display the conditional tables referred to in Proposition 3.4

1. List of the 24 tables with 1 and 2 as designated values:

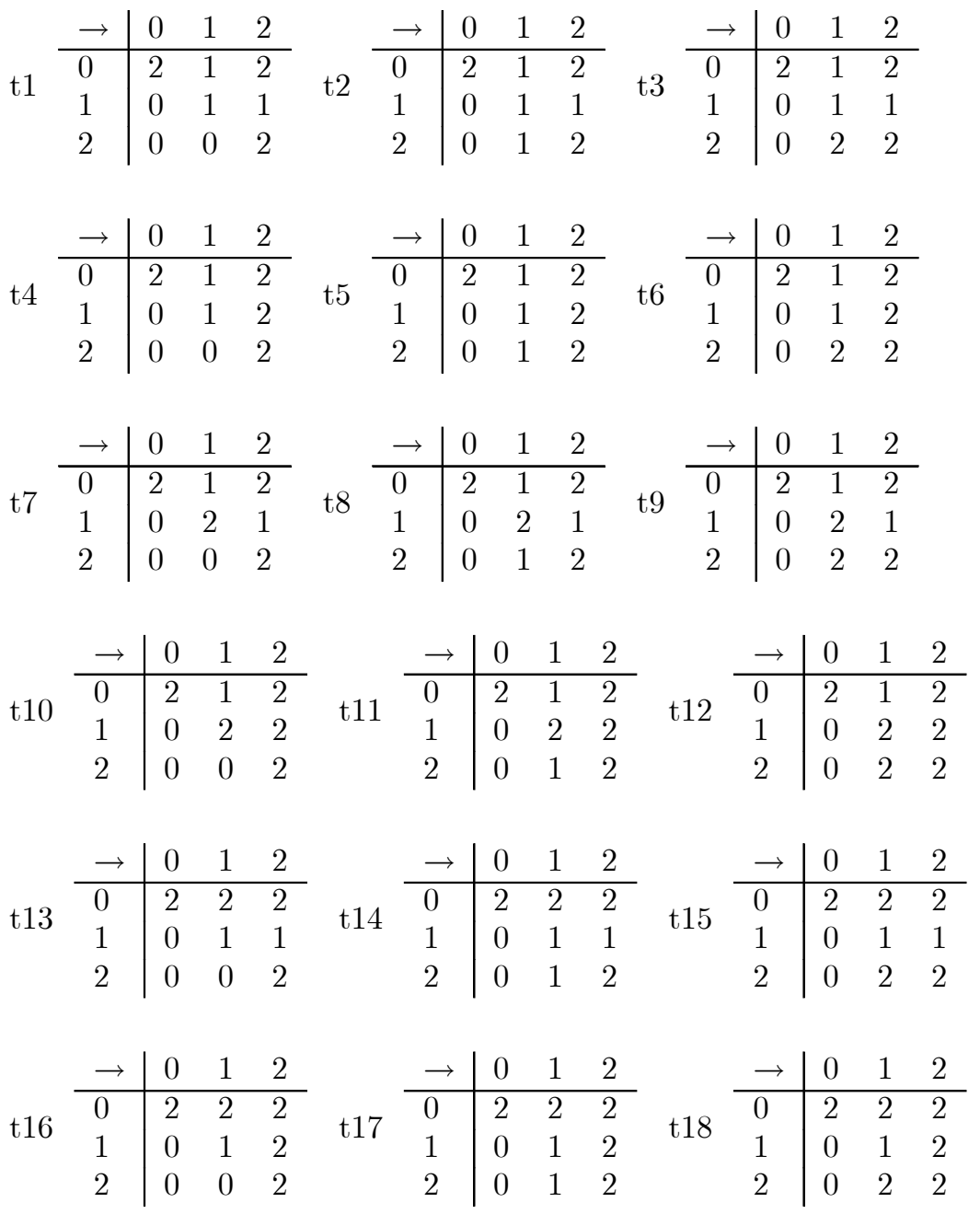




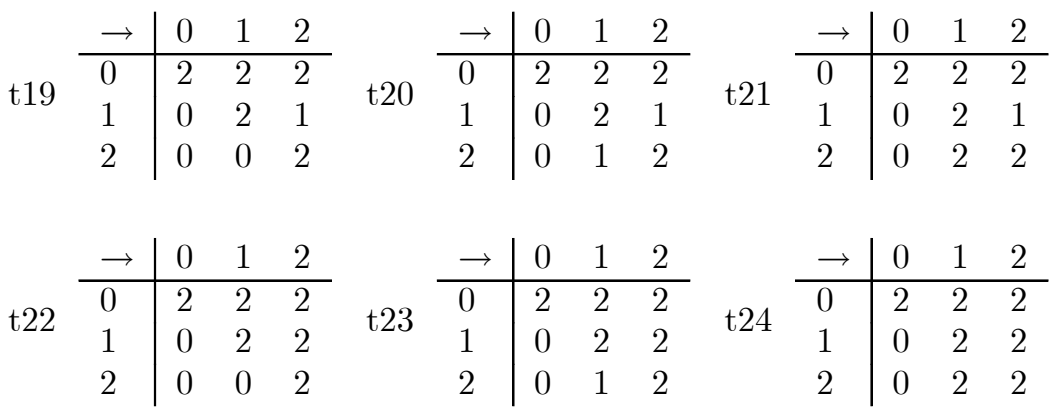

2. List of the 6 tables with 2 as the only designated value:

\begin{tabular}{|c|c|c|c|c|c|c|c|c|c|c|c|c|c|}
\hline \multirow{4}{*}{$\mathrm{t} 25$} & 0 & 1 & 2 & \multirow{4}{*}{ t26 } & $\rightarrow$ & 0 & 1 & 2 & \multirow{4}{*}{ t27 } & $\rightarrow$ & 0 & 1 & 2 \\
\hline & 2 & 2 & 2 & & 0 & 2 & 2 & 2 & & 0 & 2 & 2 & 2 \\
\hline & 2 & 2 & 2 & & 1 & 2 & 2 & 2 & & 1 & 0 & 2 & 2 \\
\hline & 0 & 1 & 2 & & 2 & 0 & 0 & 2 & & 2 & 0 & 0 & 2 \\
\hline \multirow{4}{*}{ t28 } & 0 & 1 & 2 & \multirow{4}{*}{ t29 } & $\rightarrow$ & 0 & 1 & 2 & \multirow{4}{*}{ t30 } & $\rightarrow$ & 0 & 1 & 2 \\
\hline & 2 & 2 & 2 & & 0 & 2 & 2 & 2 & & 0 & 2 & 2 & 2 \\
\hline & 1 & 2 & 2 & & 1 & 0 & 2 & 2 & & 1 & 1 & 2 & 2 \\
\hline & 0 & 1 & 2 & & 2 & 0 & 1 & 2 & & 2 & 0 & 0 & 2 \\
\hline
\end{tabular}

\section{B Appendix 2. The Lt $i$-logics as axiomatized in [14] and [15]}

In [14] and [15], the Lti-logics Lt1, Lt2,.., Lt6 are axiomatized as follows.

1. Common axioms to Lt1-Lt6:

$$
\begin{aligned}
& \text { a1. }(A \wedge B) \rightarrow A /(A \wedge B) \rightarrow B \\
& \text { a2. }[(A \rightarrow B) \wedge(A \rightarrow C)] \rightarrow[A \rightarrow(B \wedge C)] \\
& \text { a3. } A \rightarrow(A \vee B) / B \rightarrow(A \vee B) \\
& \text { a4. }[(A \rightarrow C) \wedge(B \rightarrow C)] \rightarrow[(A \vee B) \rightarrow C] \\
& \text { a5. }[A \wedge(B \vee C)] \rightarrow[(A \wedge B) \vee(A \wedge C)] \\
& \text { a6. } \neg(A \vee B) \leftrightarrow(\neg A \wedge \neg B) \\
& \text { a7. } \neg(A \wedge B) \leftrightarrow(\neg A \vee \neg B) \\
& \text { a8. } A \leftrightarrow \neg \neg A \\
& \text { a9. }[\neg(A \rightarrow B) \wedge \neg A] \rightarrow A \\
& \text { a10. }[\neg(A \rightarrow B) \wedge B] \rightarrow \neg B \\
& \text { a11. }(\neg A \wedge B) \rightarrow(A \rightarrow B)
\end{aligned}
$$


2. Common rules to Lt1-Lt6:

Adjunction (Adj): $A \& B \Rightarrow A \wedge B$

Modus Ponens (MP): $A \rightarrow B$ \& $A \Rightarrow B$

3. Common axioms to Lt5 and Lt6:

$$
\begin{aligned}
& \text { a12. } A \vee \neg A \\
& \text { a13. }[(A \rightarrow B) \wedge(B \rightarrow C)] \rightarrow(A \rightarrow C) \\
& \text { a14. }[(A \rightarrow B) \wedge A] \rightarrow B \\
& \text { a15. } A \vee(A \rightarrow B) \\
& \text { a16. }[(A \rightarrow B) \wedge \neg B] \rightarrow \neg A \\
& \text { a17. } \neg B \vee(A \rightarrow B)
\end{aligned}
$$

4. Axioms for Lt5 and Lt6:

Lt5:

a18. $(A \wedge \neg B) \rightarrow \neg(A \rightarrow B)$

Lt6:

a19. $A \rightarrow[B \vee \neg(A \rightarrow B)]$

a20. $\neg B \rightarrow[\neg A \vee \neg(A \rightarrow B)]$

a21. $[\neg(A \rightarrow B) \wedge(\neg A \wedge B)] \rightarrow C$

5. Common axioms to Lt1, Lt2, Lt3 and Lt4:

$$
\begin{aligned}
& \text { a22. }[(A \rightarrow B) \wedge A] \rightarrow(\neg A \vee B) \\
& \text { a23. }[(A \rightarrow B) \wedge \neg B] \rightarrow(\neg A \vee B) \\
& \text { a24. } \neg A \rightarrow[A \vee(A \rightarrow B)] \\
& \text { a25. } B \rightarrow[\neg B \vee(A \rightarrow B)] \\
& \text { a26. }(A \vee \neg B) \vee(A \rightarrow B)
\end{aligned}
$$

6. Common rules to Lt1, Lt2, Lt3 and Lt4:

$$
\begin{aligned}
\text { dMP: } C \vee A \& C \vee(A \rightarrow B) \Rightarrow C \vee B \\
\text { dTrans: } D \vee(A \rightarrow B) \& D \vee(B \rightarrow C) \Rightarrow D \vee(A \rightarrow C)
\end{aligned}
$$$$
\text { dEcq: } C \vee(A \wedge \neg A) \Rightarrow C \vee B
$$

(In addition to Adj and MP.) 
7. Axioms for Lt1, Lt2, Lt3 and Lt4:

Lt1:

$$
\begin{aligned}
& \text { a19. } A \rightarrow[B \vee \neg(A \rightarrow B)] \\
& \text { a20. } \neg B \rightarrow[\neg A \vee \neg(A \rightarrow B)] \\
& \text { a27. } \neg(A \rightarrow B) \rightarrow(A \vee \neg B)
\end{aligned}
$$

Lt2:

$$
\begin{aligned}
& \text { a28. } \neg(A \rightarrow B) \rightarrow(A \wedge \neg B) \\
& \text { a29. }(A \wedge \neg B) \rightarrow[\neg A \vee \neg(A \rightarrow B)]
\end{aligned}
$$

Lt3:

$$
\begin{aligned}
& \text { a20. } \neg B \rightarrow[\neg A \vee \neg(A \rightarrow B)] \\
& \text { a30. } \neg(A \rightarrow B) \rightarrow \neg B
\end{aligned}
$$

Lt4:

$$
\begin{aligned}
& \text { a19. } A \rightarrow[B \vee \neg(A \rightarrow B)] \\
& \text { a31. } \neg(A \rightarrow B) \rightarrow A
\end{aligned}
$$

Now, for $i(1 \leq i \leq 6)$, let us refer by Lt $i$ to the logic defined in section 4 and by Lt $i^{\prime}$ to the one defined in the present appendix. We have:

Proposition B.1 (Lt $i$ and Lt $i^{\prime}$ are equivalent) For $i(1 \leq i \leq 6)$, Lti and Lti' are deductively equivalent.

Proof. (a) Lt $i^{\prime}$ is deductively included in Lt $i$ : We remark the following facts. (1) a1-a8, Adj, MP, dMP and dTrans are provable in $\mathrm{B}^{\mathrm{d}}$; (2) the following are provable in $\mathrm{b}^{3}$ : $\mathrm{a} 9$, a10 (T5 and T8 in Proposition 4.8, respectively), a24 and a25 (A9 and A10, respectively); (3) the following are provable in $\mathrm{b}_{1}^{3}$ : a22, a23, a26, a29 and Ecq (Definition 4.9 and Proposition 4.10); on the other hand, notice that a11 is provable in Lt1-Lt6 (cf. Definition 4.9 and Definition 4.11); (4) the following are provable in $\mathrm{b}_{2}^{3}$ : a11-a17, a19 and a20 (Definition 4.9, Proposition 4.10). Then, it follows by inspection of the axiomatization of Lt $i$ and Lt $i^{\prime}$ that all axioms and rules of Lt $i^{\prime}$ are provable in Lti.

(b) Lti is deductively included in Lti': All axioms and rules of Lt $i$ are verified by Mti (the reader can use the tester in [9], if needed). But in [14] and [15], it is proved that Lt $i^{\prime}$ is determined by Mti (cf. Definition 2.7). Consequently, it follows from (a) above and this fact that Lt $i$ is also determined by Mti. Thus, Lt $i$ is deductively included in Lt $i^{\prime}$. 


\section{Appendix 3. Alternative axiomatizations of the Lt $i$-logics}

It is possible to give more conspicuous axiomatizations of the Lt $i$-logics. Consider the following system TR (Ticket Entailment — cf. [1] — minus the reductio axiom $(A \rightarrow \neg A) \rightarrow \neg A)$ :
b1. $A \rightarrow A$
b2. $(A \wedge B) \rightarrow A /(A \wedge B) \rightarrow B$
b3. $[(A \rightarrow B) \wedge(A \rightarrow C)] \rightarrow[A \rightarrow(B \wedge C)]$
b4. $A \rightarrow(A \vee B) / B \rightarrow(A \vee B)$
b5. $[(A \rightarrow C) \wedge(B \rightarrow C)] \rightarrow[(A \vee B) \rightarrow C]$
b6. $[A \wedge(B \vee C)] \rightarrow[(A \wedge B) \vee(A \wedge C)]$
b7. $(A \rightarrow B) \rightarrow[(B \rightarrow C) \rightarrow(A \rightarrow C)]$
b8. $[A \rightarrow(A \rightarrow B)] \rightarrow(A \rightarrow B)$
b9. $(A \rightarrow \neg B) \rightarrow(B \rightarrow \neg A)$
b10. $(\neg A \rightarrow B) \rightarrow(\neg B \rightarrow A)$

The only rules of inference are Adj and MP.

Consider now the result of extending TR with the following axioms:

$$
\begin{aligned}
& \text { b11. } A \rightarrow(A \rightarrow A) \\
& \text { b12. } \neg A \rightarrow[A \vee(A \rightarrow B)] \\
& \text { b13. }(\neg A \wedge B) \rightarrow(A \rightarrow B) \\
& \text { b14. } A \vee \neg A
\end{aligned}
$$

Let us name eTR this extension of TR. Then, the Lti-logics are axiomatized as follows.

- Lt5: eTR \& $(A \wedge \neg B) \rightarrow \neg(A \rightarrow B)$ (b15).

- Lt6: eTR \& $[\neg(A \rightarrow B) \wedge(\neg A \wedge B)] \rightarrow C$ (b16).

- Lt1: Lt6 minus b14 plus $(A \vee \neg B) \vee(A \rightarrow B)$ (b17) and dEcq.

- Lt2: b1-b7, b9, b10, b17, $A \rightarrow(B \rightarrow A)(\mathrm{b} 18),[(A \rightarrow B) \wedge A] \rightarrow(\neg A \vee B)$ (b19), Adj, MP, dMP and dEcq.

- Lt3: b1-b8, b12, b17, b18, $A \rightarrow \neg \neg A(\mathrm{~b} 20), \neg \neg A \rightarrow A(\mathrm{~b} 21),[(A \rightarrow$ $B) \wedge \neg B] \rightarrow(\neg A \vee B)(\mathrm{b} 22), B \rightarrow[\neg B \vee(A \rightarrow B)]$ (b23), Adj, MP, dCon and dEcq.

- Lt4: $\mathrm{b}^{3}$ Ecq \& b17, b19, $[(A \rightarrow B) \wedge \neg B] \rightarrow \neg A(\mathrm{~b} 24)$ and $\neg A \rightarrow(A \rightarrow B)$ (b25). 
It would not be difficult to prove that the axiomatizations of the Lti-logics given above are (deductively) equivalent to the ones discussed in Appendix 2, but we do not have space to develop this point here.

Funding. This work was supported by the Spanish Ministry of Economy, Industry and Competitiveness [FFI2014-53919-P, FFI2017-82878-P].

Acknowledgements. I sincerely thank two anonymous referees of the IGPL for their comments and suggestions on a previous version of this paper.

\section{References}

[1] Anderson, A. R., Belnap, N. D. Jr. (1975). Entailment. The Logic of Relevance and Necessity, vol I. Princeton, NJ: Princeton University Press.

[2] Avron, A. (1991). Natural 3-Valued Logics-Characterization and Proof Theory. Journal of Symbolic Logic, 56(1), 276-294.

[3] Belnap, N. D. Jr. (1977). How a computer should think. In G. Ryle (Ed.), Contemporary Aspects of Philosophy (pp. 30-55). Oriel Press Ltd., Stocksfield.

[4] Belnap, N. D. J.r (1977). A useful four-valued logic. In G. Epstein \& J. M. Dunn (Eds.), Modern Uses of Multiple-Valued Logic (pp. 8-37). D. Reidel Publishing Co., Dordrecht.

[5] Brady, R. T. (ed.) (2003). Relevant Logics and Their Rivals, vol. II. Ashgate, Aldershot.

[6] Brady, R. T. (1982). Completeness Proofs for the Systems RM3 and BN4. Logique et Analyse, 25, 9-32.

[7] Dunn, J. M. (1976). Intuitive semantics for first-degree entailments and "coupled trees." Philosophical Studies, 29, 149-168.

[8] Dunn, J. M. (2000). Partiality and its Dual. Studia Logica, 65, 5-40. http://doi.org/10.1023/A:1026740726955.

[9] González, C. (2012). MaTest. Retrieved from http://ceguel.es/matest (last accessed 24/02/2018).

[10] Kleene, S. C. (1952). Introduction to Metamathematics. North Holland. Reprinted Ishi Press, 2009.

[11] Robles, G. (2013). A Routley-Meyer semantics for Gödel 3-valued logic and its paraconsistent counterpart. Logica Universalis, 7(4), 507-532, doi: $10.1007 / \mathrm{s} 11787-013-0088-7$. 
[12] Robles, G., Méndez, J. M. (2014). A Routley-Meyer semantics for truthpreserving and well-determined Łukasiewicz 3-valued logics. Logic Journal of the IGPL, 22(1), 1-23, doi: 10.1093/jigpal/jzt017.

[13] Robles, G., Blanco, J. M., López, S. M., Paradela, J. R., Recio, M. M. (2016). Relational semantics for the 4-valued relevant logics BN4 and E4. Logic and Logical Philosophy, 25, 173-201, doi: 10.12775/LLP.2016.006.

[14] Robles, G., Méndez, J. M. (Submitted). Belnap-Dunn semantics for natural implicative expansions of Kleene's strong three-valued matrix with two designated values.

[15] Robles, G., Salto, F., Méndez, J. M. (Submitted). Belnap-Dunn semantics for natural implicative expansions of Kleene's strong three-valued matrix II. Only one designated value.

[16] Routley, R. Meyer, R. K., Plumwood, V., Brady R. T. (1982). Relevant Logics and their Rivals, vol. 1. Atascadero, CA: Ridgeview Publishing Co.

[17] Sylvan, R., Plumwood, V. (2003). Non-normal relevant logics. In R. . Brady (Ed.), Relevant logics and their rivals, vol. II (pp. 10-16). Ashgate, Aldershot and Burlington: Western Philosophy Series.

[18] Tomova, N. (2012). A Lattice of implicative extensions of regular Kleene's logics. Reports on Mathematical Logic, 47, 173-182. http://doi.org/10.4467/20842589RM.12.008.0689 